\title{
Two different types of amyloid deposits-apolipoprotein A-IV and transthyretin-in a patient with systemic amyloidosis
}

Joakim Bergström ${ }^{1}$, Charles L Murphy², Deborah T Weiss ${ }^{2}$, Alan Solomon ${ }^{2}$, Knut Sletten ${ }^{3}$, Ulf Hellman ${ }^{4}$ and Per Westermark ${ }^{1}$

${ }^{1}$ Department of Genetics and Pathology, Uppsala University, Uppsala, Sweden; ${ }^{2}$ Human Immunology and Cancer Program, Department of Medicine, University of Tennessee Graduate School of Medicine, Knoxville, TN, USA; ${ }^{3}$ Department of Biochemistry/Biotechnology Centre of Oslo, University of Oslo, Norway and

${ }^{4}$ Ludwig Institute for Cancer Research, Uppsala, Sweden

\begin{abstract}
Certain forms of systemic amyloidosis have been associated with the pathologic deposition as fibrils of three different apolipoprotein-related proteins-apolipoprotein A-I, apolipoprotein A-II, and serum amyloid A. We have previously reported (Bergström et al, Biochem Biophys Res Commun 2001;285:903-908) that amyloid fibrils extracted from the heart of an elderly male with senile systemic amyloidosis contained, in addition to wild-type transthyretin-related molecules, an N-terminal fragment of yet a fourth apolipoprotein-apolipoprotein A-IV (apoA-IV). We now provide the results of our studies that have established the complete amino-acid sequence of this $\sim 70$-residue component and, additionally, have shown this protein to be the product of an unmutated apoA-IV gene. Notably, the apoA-IV and transthyretin fibrils were not codeposited but, rather, had anatomically distinct patterns of distribution within the heart and other organs, as evidenced immunohistochemically, by variation in the ultra structural morphology and by differences in the intensity of Congo red birefringence. These findings provide the first conclusive evidence that two separate forms of amyloid, each derived from a wild-type amyloidogenic precursor protein, were present in a patient with systemic amyloidosis. Laboratory Investigation (2004) 84, 981-988, advance online publication, 17 May 2004; doi:10.1038/labinvest.3700124
\end{abstract}

Keywords: amyloidosis; apolipoprotein A-IV; fibril; systemic; transthyretin

The systemic amyloidoses comprise a group of often life-threatening protein conformational diseases that are associated with the pathologic deposition as fibrils of at least 12 structurally diverse molecules in vital organs and tissues throughout the body. ${ }^{1}$ Most of these proteins are chemically and structurally unrelated, ${ }^{2}$ yet the abnormal product, the amyloid fibril, exhibits virtually identical tinctorial and ultra structural features. ${ }^{3}$

The amyloid fibril core has a high degree of $\beta$ sheet structure, with the $\beta$-strands configured in an orientation perpendicular to the fibril axis and with hydrogen bonds formed between monomeric subunits. ${ }^{4}$ Many of the amyloidogenic precursor pro-

Correspondence: Dr P Westermark, MD, PhD, Department of Genetics and Pathology, Uppsala University, Rudbeck Laboratory, 75185 Uppsala, Sweden.

E-mail: per.westermark@genpat.uu.se

Received 8 March 2004; revised and accepted 5 April 2004; published online 17 May 2004 teins also display extensive $\beta$-structure in their natively folded form (eg, transthyretin, $\beta 2$-microglobulin and immunoglobulin light chains), but in other cases (eg, apolipoprotein A-I (apoA-I) and apolipoprotein A-II (apoA-II)), an $\alpha$-helix to $\beta$-sheet transition must occur. ${ }^{5}$ Additionally, some proteins that are natively unfolded also may adopt a $\beta$ conformation and form amyloid fibrils. ${ }^{6}$

Transthyretin (TTR) is the protein found in most types of familial amyloidosis. In these diseases, a missense mutation in the TTR gene results in an amino-acid substitution at a particular position in this 127-residue component; notably, more than 85 different alterations have been deemed amyloidogenic. ${ }^{7}$ The clinical phenotypes vary, but polyneuropathy and cardiomyopathy predominate. TTR also comprises the pathologic protein found in senile systemic amyloidosis (SSA) where the amyloid fibrils are composed predominately of C-terminal fragments of wild-type (wt) TTR that start at positions 46,49 , and $52 .{ }^{8} \mathrm{SSA}$ is probably the most 
common of all systemic amyloidoses and in autopsy studies has been seen in 18-25\% of individuals over the age of 80 years. ${ }^{9-11}$ However, in a Japanese study, only $8 \%$ of individuals 80 -years old and over had TTR-derived amyloidosis. ${ }^{12}$ In most cases, the fibrillar deposits are small, affecting blood vessels in different organs and, seemingly, are not of pathophysiological import. However, in some individuals, mainly elderly males, extensive cardiac deposition of wt TTR can occur that results in cardiomegaly and eventual death due to heart failure. ${ }^{13,14}$ Such patients also may have pronounced pulmonary amyloid infiltration, as well as involvement of the renal papillae. ${ }^{14}$ Among other proteins associated with the systemic amyloidoses, the highdensity apolipoproteins are of special interest, for example, apoA-I and apoA-II that constitute the fibrils found in several familial forms of this disorder. $^{5,15-20}$ Another, serum amyloid A, is the precursor protein of fibrils in secondary or reactive (AA) amyloidosis. ${ }^{21}$ Why this class of molecules is prone to aggregate and form amyloid fibrils is not known. However, our finding of yet another amyloid-associated apolipoprotein, namely, apolipoprotein A-IV (apoA-IV), ${ }^{22}$ suggests that these components share structural features that render them amyloidogenic.

Among individual patients with systemic amyloidosis, the amyloid typically is composed of only one type of protein, although minor populations of other molecules, for example, apolipoprotein E, also may be present in the deposits. ${ }^{23}$ However, previously we had identified, through chemical and immunologic analyses of fibrils extracted from the heart of an elderly male who had clinical and pathologic features characteristic of SSA, both apoA-IV and wt TTR. ${ }^{22}$ The unexpected detection of apoA-IV provided the first example that this apolipoprotein could be amyloidogenic. We had posited that the two different amyloids were codeposited, based on our demonstration that synthetic apoA-IV polypeptides were inherently fibrillogenic and could serve as seeds to promote in vitro TTR fibril formation. ${ }^{22}$

We now report the results of our latest studies where the entire primary structure of the apoA-IVderived amyloid protein was established. Additionally, it was determined that this component, like the TTR, was a product of an unmutated gene. Most notable was our finding that the apoA-IV and TTR fibrils were not codeposited but, instead, occurred separately within the heart and other organs. Thus, our patient seemingly had two independent systemic amyloid diseases.

\section{Materials and methods}

\section{Tissue Specimens}

Specimens of heart and other tissues obtained at autopsy from a 92-year-old male with pronounced cardiac amyloidosis (heart weight, 620 g) were frozen and preserved at $-25^{\circ} \mathrm{C}$ and stored for 4 years prior to fibril extraction. Tissue specimens were also fixed in $4 \%$ buffered neutral formaldehyde solution and embedded in paraffin.

\section{Immunohistochemistry and Amyloid Identification}

Two polyclonal anti-apoA-IV antisera were prepared by immunizing rabbits with a keyhole limpet hemocyanine-linked synthetic peptide corresponding to positions $44-55$ of apoA-IV. The antisera were specific for apoA-IV, as evidenced in an enzymelinked immunosorbent assay (ELISA). Rabbit antiserum to TTR was raised against an in vitro expressed protein fragment, corresponding to positions 50-127 of human TTR, coupled to keyhole limpet hemocyanine. This reagent was found to label TTR-amyloid specifically and did not crossreact with apoA-IV or other known amyloidogenic proteins. Deparaffinized $5 \mu \mathrm{m}$-thick sections were incubated overnight with antibody diluted 1:3000$1: 8000$, washed, and the immunoreactivity visualized using the biotin/streptavidin method with $3,3^{\prime}$ diaminobenzidine-tetrahydrochloride as substrate. Immunostained sections also were treated with an alkaline Congo red solution and examined under polarized light for simultaneous demonstration of amyloid birefringence and immunoreactivity. ${ }^{24,25}$

\section{Amyloid Isolation, Purification, and Characterization}

Amyloid protein was extracted from fresh-frozen heart and defatted. ${ }^{8}$ Fibrils were dissolved in $6 \mathrm{M}$ guanidine $\mathrm{HCl} / 0.1 \mathrm{M}$ Tris-HCl, $\mathrm{pH}$ 8.0, containing $0.1 \mathrm{M}$ dithiothreitol. After incubation at room temperature for $48 \mathrm{~h}$, the fibril solution was centrifuged, after which the supernatant was dialyzed against saturated ammonium sulfate followed by deionized water, and then freeze-dried. The lyophilized material was redissolved in $6 \mathrm{M}$ guanidine $\mathrm{HCl} / 0.1 \mathrm{M}$ Tris-HCl, pH 8.0 containing $0.1 \mathrm{M}$ dithiothreitol and applied to a $1.6 \times 90 \mathrm{~cm}$ Sepharose 6B-CL column (Amersham Biosciences, Uppsala, Sweden) equilibrated with $5 \mathrm{M}$ guanidine $\mathrm{HCl}$ (flow rate, $4 \mathrm{ml} / \mathrm{h}$ ) and the absorbance monitored at $280 \mathrm{~nm}$.

In order to map the heart for areas with apoA-IV amyloid deposits, small pieces of fresh frozen cardiac tissue were taken from six different anatomical sites. This material was squeezed between glass microscopic slides, dried, and the apoA-IV identified immunohistochemically. Based on these results, a $4 \mathrm{~g}$ portion was taken from the part of the heart that showed the most pronounced apoA-IV reactivity and the amyloid protein was extracted as described above.

\section{Enzyme-Linked Immunosorbent Assay}

The apoA-IV-contained in gel filtration fractions was identified by ELISA. A volume of $50-\mu \mathrm{l}$ aliquots from each fraction were diluted to $150 \mu \mathrm{l}$ with $50 \mathrm{mM}$ 
sodium carbonate buffer, $\mathrm{pH}$ 9.6, containing $0.2 \%$ sodium azide and coated onto a 96-well Immulon 2 HB polystyrene microtiter plate (Dynex Laboratories, Alexandria, VA, USA). After washing, the ELISA plate was incubated overnight at room temperature with the anti-apoA-IV antiserum diluted 1:100. Alkaline phosphatase-conjugated goat anti-rabbit immunoglobulin antiserum (Dako, Glostrup, Denmark) was used as the secondary antibody at a dilution of 1:1500. Immunoreactivity was visualized using $p$-nitrophenyl-phosphate (Sigma, St Louis, MO, USA).

\section{Reverse-Phase HPLC}

The gel-filtration fractions containing the highest apoA-IV reactivity (as determined by ELISA), were subjected to reverse-phase HPLC (RP-HPLC) on a Brownlee Aquapore BU-300 $30 \times 4.6 \mathrm{~mm}$ C4 column (Perkin-Elmer, Norwich, CT, USA) using a gradient of $0-70 \%$ acetonitrile in $0.1 \%$ trifluoroacetic acid at a flow rate of $0.4 \mathrm{ml} / \mathrm{min}$; the absorbance was monitored at $220 \mathrm{~nm}$ and the peaks collected manually. Aliquots $(25 \mu \mathrm{l})$ from each were diluted with $50 \mathrm{mM}$ sodium carbonate buffer, $\mathrm{pH}$ 9.6, containing $0.2 \%$ sodium azide to a total volume of $100 \mu \mathrm{l}$ and analyzed for apoA-IV content by ELISA. The apoA-IV-containing fractions were vacuumdried or concentrated using a Speed Vac (Savant Instruments, Farmingdale, NY, USA).

\section{Amino-Acid Sequencing and Mass Spectrometry}

N-terminal amino-acid sequence analysis of RPHPLC-purified, intact apoA-IV protein by direct Edman degradation was performed as previously described. ${ }^{26}$ This material was also digested with trypsin or endoproteinase Asp- $\mathrm{N}^{8,22}$ (Roche Applied Sciences, Germany) and the resulting peptides subjected to amino-acid sequencing or mass spectrometry $^{27}$ using a matrix-assisted laser desorption/ ionization time-of-flight mass spectrometer (MALDITOF) (Ultraflex TOF/TOF, Bruekr Daltonics, Bremen, Germany).

\section{Western Blot Analysis}

Amyloid fibrils were dissolved in $6 \mathrm{M}$ guanidine/ $0.1 \mathrm{M}$. Tris-HCl, pH 8.0, dialyzed, and lyophilized as described above. This material, as well as protein isolated by RP-HPLC, was dissolved in sample buffer containing $3 \%$ sodium dodecyl sulfate (SDS) and electrophoresed on SDS-PAGE gels. ${ }^{28}$ Proteins were blotted onto a $0.3 \mu \mathrm{m}$ nitrocellulose membrane (Amersham Biosciences, Uppsala, Sweden) and exposed first to the anti-apoA-IV antiserum (1:500 dilution) and then to a secondary antibody (horse radish peroxidase-conjugated swine antirabbit immunoglobulin (Dako, Glostrup, Denmark) diluted 1:10 000. The reaction was visualized using an enhanced chemiluminescence system (Amersham Biosciences, Uppsala, Sweden).

\section{Electron Microscopy}

A small piece of heart from the upper dorsal wall of the left ventricle that had both apoA-IV and TTR immunoreactive deposits (as demonstrated immunohistochemically) was fixed in a solution containing $0.25 \%$ glutaraldehyde and $2 \%$ paraformaldehyde in $0.1 \mathrm{M}$ sodium phosphate buffer, $\mathrm{pH} 7.4$, and embedded in Unicryl (British BioCell, Cardiff, UK). Ultra thin sections were placed on formvar-coated nickel grids and immunolabeled with the anti-TTR or anti-apoA-IV antisera diluted 1:200. Immunoreactivity was demonstrated with goat anti-rabbit antibodies labeled with $10-\mathrm{nm}$ gold particles (British Biocell, Cardiff, UK).

\section{Genetic Analysis}

DNA was extracted from fresh-frozen heart using the Genomic Prep Cell and Tissue DNA Isolation kit (Amersham Biosciences, Uppsala, Sweden). Exons 1,2 , and 3 of the apoA-IV gene were amplified by PCR with the following primers: 5'-TGTGGCAA GAAACTCCTCCA- $3^{\prime}$ and $5^{\prime}$-AGTGCCATCCAAAGA CAGCTT-3' (exon 1); 5'-CATCATCCAGTCTGCAGC TCA-3' and $5^{\prime}$-CGTACATTGCATGGCCT TT-3' (exon 2); 5'-CTTGCCGTGTAAATGCCAAA-3' ${ }^{\prime}, 5^{\prime}$-TTCTCC CGCAGCACTCTCT-3' ${ }^{\prime}$, 5'-TAGCACAGCGCATGGAG AGA-3' ${ }^{\prime}$, 5'-AGTGACTTCTGCAGCCCT-3' ${ }^{\prime}, 5^{\prime}$-TTCCA GAATGAAGAAGAACGCC-3' ${ }^{\prime}$, 5'-AGGAGTTGACCT TGTCCCTCA-3, 5'-AACAGCTCAAGGCAGAAACTG G-3' and $5^{\prime}$-AAGGAGGATTCATCCGGCAA- $3^{\prime}$ (exon 3). The DNA was dissolved in a $1 \times$ PCR buffer (15 mM Tris/HCl, pH 8.0), containing $50 \mathrm{mM} \mathrm{KCl,}$ $2.5 \mu \mathrm{M} \mathrm{MgCl}_{2}, 200 \mu \mathrm{MdNTPs}, 1.25 \mathrm{U}$ AmpliTaq Gold ${ }^{\circledR}$ DNA Polymerase (Applied Biosystems, Foster City, CA, USA) and $0.4 \mu \mathrm{M}$ of each of the sense and antisense primers. The 30-cycle protocol included: $5 \mathrm{~min}$ initial heating at $95^{\circ} \mathrm{C}, 30 \mathrm{~s}$ denaturation at $95^{\circ} \mathrm{C}, 30 \mathrm{~s}$ annealing at $59^{\circ} \mathrm{C}$, and an 1 min extension at $72^{\circ} \mathrm{C}$. The final extension was performed for $7 \mathrm{~min}$ at $72^{\circ} \mathrm{C}$. PCR products were separated by electrophoresis on a $1.5 \%$ agarose gel and the bands were excised and purified using a Qiaex II gel extraction kit (Qiagen, Valencia, CA, USA) and sequenced (Kiseq, Karolinska Institute, Stockholm, Sweden).

\section{Results}

\section{Chemical Nature of the ApoA-IV Fibrils}

Amyloid fibrils were extracted from a 4-g portion of fresh frozen heart tissue that contained the most pronounced apoA-IV reactivity, as evidenced immunohistochemically. Western blotting analysis (Figure 1) revealed the presence of an apoA-IVrelated component with a molecular mass of $\sim 8 \mathrm{kDa}$, a value considerably less than that of the 


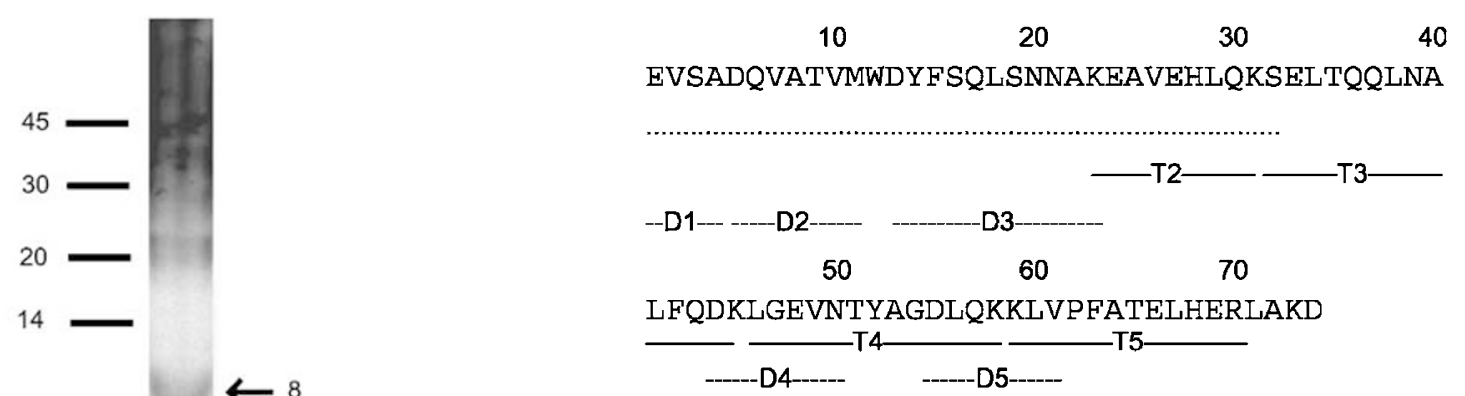

Figure 1 Characterization of apoA-IV amyloid fibrils. The protein extracted from cardiac amyloid deposits was electrophoresed on an SDS-PAGE gel and immunoblotted with a specific anti-apoAIV antiserum. The molecule weight markers and location of the $\sim 8 \mathrm{kDa}$ component are as indicated.

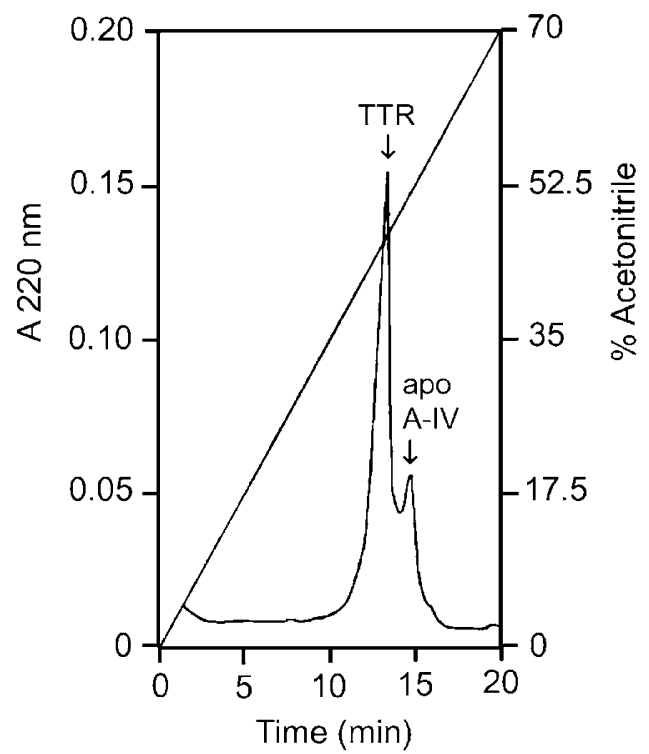

Figure 3 Primary structure of the $\sim 8 \mathrm{kDa}$ apoA-IV amyloid protein. The sequence of this 70-residue component was determined directly from the intact component $(\cdots)$ and from Asp-N (D) and tryptic (T) peptides, as indicated.

apoA-IV containing amyloid extracted from formalin-fixed, paraffin-embedded tissue sections. ${ }^{22}$ Through MALDI-TOF spectrometry of trypsindigested RP-HPLC-purified material, residues located between positions 23 and 70 were identified (Figure 3). Together, these data established that the apoA-IV-related amyloid protein represented an $\sim 70$-residue $\mathrm{N}$-terminal fragment of the mature protein and had the amino-acid sequence expected for that portion of the wt molecule.

\section{Characterization of the ApoA-IV Gene}

The cDNA encoding the three exons of the apoA-IV gene was cloned from genomic DNA. The predicted protein sequence was identical to that expected for the wt product, that is, it contained no mutations. Although, both Arg and Lys residues have been identified at position 259 in apoA-IV, ${ }^{29,30}$ we found only an Arg-specifying triplet (AGG) at this location.

\section{Distribution of ApoA-IV and TTR Amyloid}

Examination by polarizing microscopy of Congo redstained tissue sections revealed that amyloid deposits were present in the heart, lung, kidney, and seminal vesicles, while none were evident in the liver. Notably, two distinct staining patterns were seen within the heart. The first was characterized by bright and glittering birefringence appearing as small patchy deposits that appeared to be located intracellularly within cardiac myocytes as well as both interstitial deposits and larger nodular deposits (Figure $4 \mathrm{a}-\mathrm{C}$ ). This material was immunostained using the specific anti-apoA-IV antiserum (Figure $4 \mathrm{~b}$ and $\mathrm{c}$ ). In the second and more predominate pattern, the amyloid deposits were stained less intensely by Congo red and were only weakly birefringent. This material, which was evident in both interstitial deposits and in the form of larger nodules that had a patchy distribution, was immunostained by the specific anti-TTR reagent (Figure 4a and d). Furthermore, TTR immunoreactive deposits of varying size commonly were found in vessel walls. In the cardiac conduction system, apoA-IV-containing amyloid 

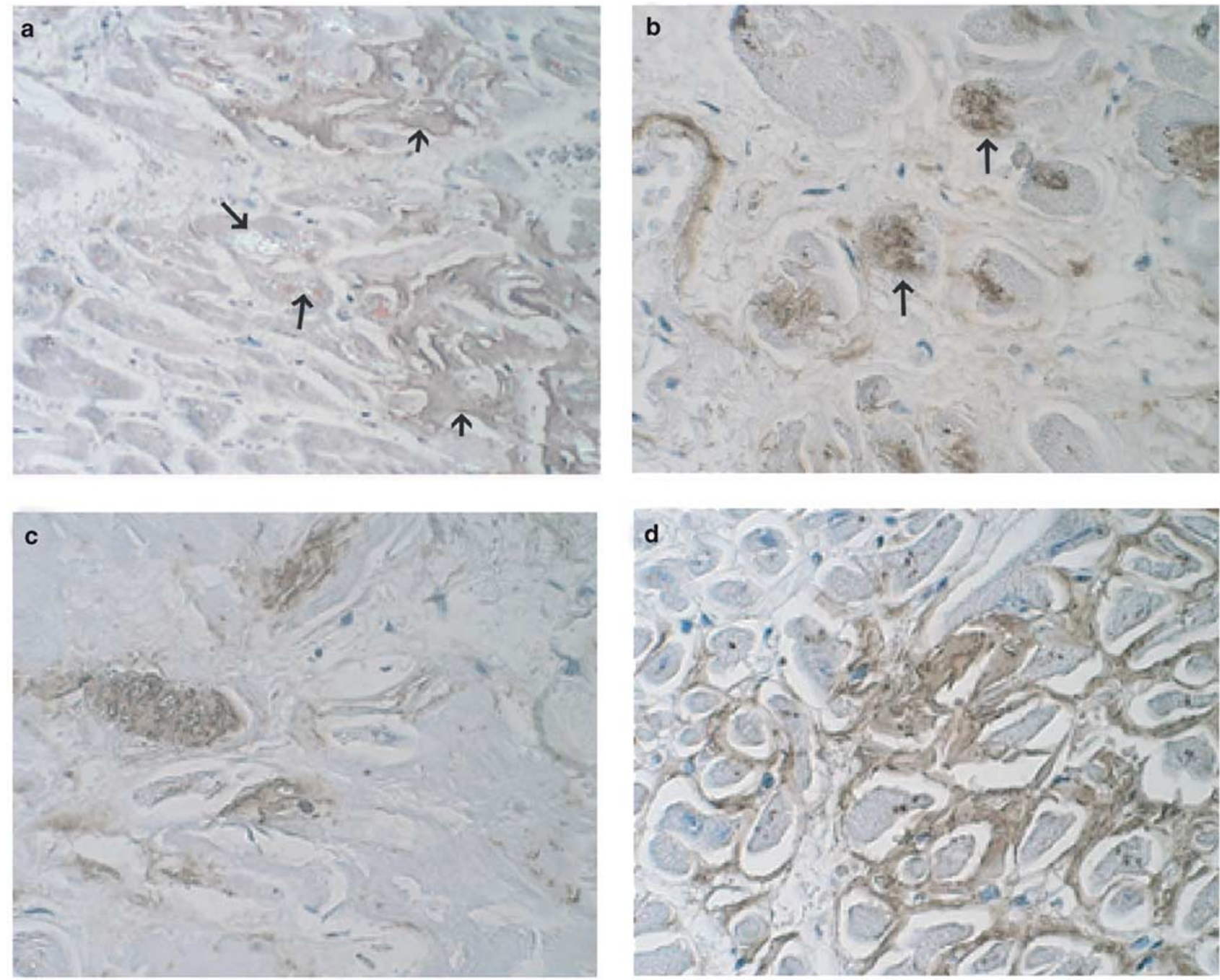

Figure 4 Immunohistochemical demonstration of TTR-and apoA-IV-related cardiac amyloid deposits. Sections in panels (a) and (d) were immunostained with anti-TTR antibodies and those in panels (b) and (c), with the anti-apoA-IV reagent. Additionally, the heart tissue in panel (a) was counterstained with Congo red and viewed under polarized light. Long arrows indicate apoA-IV amyloid deposits and short arrows ATTR-amyloid (original magnification $\times 280$ ).

was exclusively located near the atrio-ventricular node, whereas that associated with TTR was deposited adjacent to both the atrio-ventricular and the sinus nodes.

In the lung, apoA-IV amyloid occurred in vessel walls, while that derived from TTR was seen as small deposits in alveolar septa (as characteristically found in SSA). In both the seminal vesicles and kidney, anti-apoA-IV and -TTR antisera labeled anatomically distinct sites in blood vessel walls. Additionally, large amounts of TTR (but not apoAIV) amyloid were found in the renal papillae. Although both types occasionally were found in the vicinity of each other, only very rarely was apoA-IV and TTR immunoreactivity colocalized.

\section{Electron Microscopy}

The two forms of amyloid also could be distinguished ultrastructurally. As evidenced from the immunogold-labeling patterns, the anti-apoA-IV antibody reacted with bundles of parallel, relatively thick fibrils. In contrast, the anti-TTR reagent recognized more slender, nonparallel fibrils running in different directions (Figure 5).

\section{Discussion}

In this study, we show for the first time that apoA-IV is a major fibril protein in one form of systemic amyloidosis. Through amino-acid sequencing and mass spectrometric analyses, we now have shown that this amyloid mainly consisted of an $\sim 70-$ residue N-terminal fragment of apoA-IV, presumably formed through proteolytic cleavage of the native protein. Thus, apoA-IV, like the apolipoproteins serum amyloid A, apoA-I and apoA-II, can be amyloidogenic. Additionally, apolipoprotein E has been found to be a minor component in other 

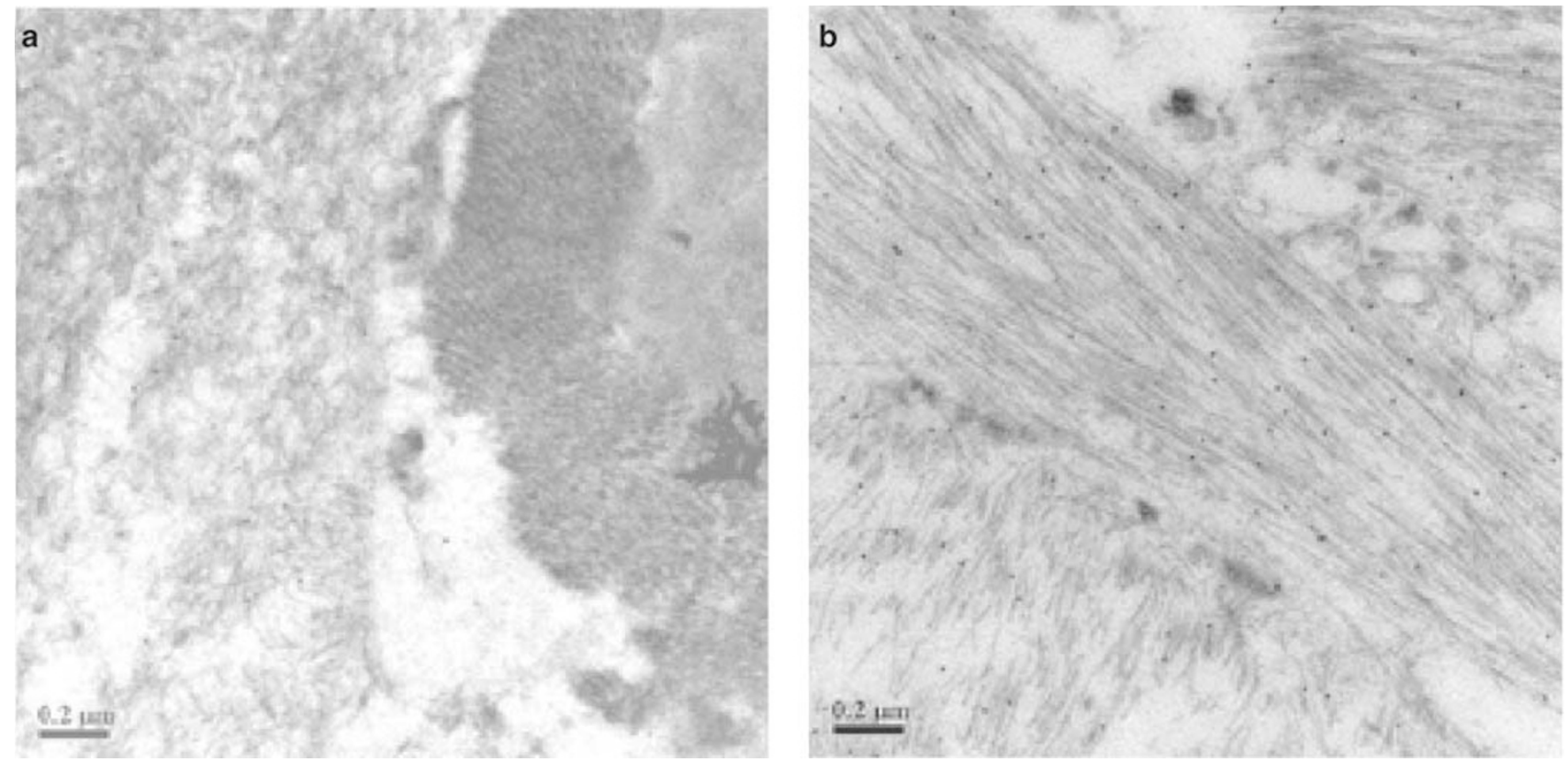

Figure 5 Ultrastructural features of apoA-IV and TTR amyloid fibrils. Electron micrographs of apoA-IV (panel b) and TTR (panel a) fibrils immunolabeled with 10-nm gold particle-conjugated anti-apoA-IV and anti-TTR antisera, respectively. Bar $=0.2 \mu \mathrm{m}$.

chemically diverse forms of amyloid. $^{23}$ ApoA-I, apoA-IV and apolipoprotein E have similar molecular structures, including $\alpha$-helix repeats of 11 or 22 amino acids, often separated by a proline residue. $^{31,32}$ These molecules have a critical role in lipid transport and metabolism and their amphipathic helices constitute the lipid binding and lecithin:cholesterol acyltransferase activating regions of the proteins. ${ }^{33,34}$ Apolipoproteins may be unusually prone to form amyloid fibrils in vivo, possibly as a result of their intrinsic flexibility. ${ }^{5}$ They have been shown to adopt a more stable conformation after binding to lipid surfaces; possibly due to a conversion of random coil structure to an $\alpha$-helical structure. ${ }^{35}$

Studies on apoA-I have indicated that cleavage of the full-length protein and generation of an $\mathrm{N}$ terminal fragment are important steps in amyloidogenesis $;{ }^{5}$ most likely, this also occurs in the case of other apolipoprotein-associated amyloidoses. For example, a portion of apoA-I, consisting of the first 93 residues, assumes a random coil configuration in water; however, when it forms fibrils, the protein first has to adopt a helical conformation in order to generate a stable $\beta$-sheet structure. ${ }^{5} \mathrm{~A}$ recent study has shown that the binding of apoA-I to lipid is a two-step process that increases the overall $\alpha$-helicity of the molecule. ${ }^{32}$ In this mechanism, which also has been proposed for apoA-IV, ${ }^{32}$ the C-terminal amphipathic helices of apoA-I initially bind to the lipid surface, which results in a major conformational change in the N-terminal part of the molecule. This leads to hydrophobic helix-lipid interactions, rather than the intramolecular N-terminal hydrophobic helix-helix interactions that would other- wise occur. ${ }^{32}$ The binding of apoA-IV to lipid surfaces also can lead to structural alterations in which hydrophobic buried N-terminal residues are unfolded and reoriented towards the lipid surface. ${ }^{35}$ This native plasticity in the N-terminal part of apoAI and apoA-IV could explain why the N-terminal part of these proteins is prone to start to aggregate. In addition, the N-terminal part of apoA-IV is the most hydrophobic part and contains one of its two native beta sheet structures, ranging from residue 6 to $15 .^{36,37}$ In the case of apoA-IV, this apolipoprotein, above all others, exhibits the highest sensitivity to guanidine hydrochloride-induced denaturation, indicating a marginal stability for its native $\alpha$-helical structure. ${ }^{38}$ Therefore, it may be particularly sensitive to environmental perturbations. Furthermore, apoA-IV is the most hydrophilic apolipoprotein and has the lowest affinity for lipid surfaces. ${ }^{36}$

Most forms of apolipoprotein-associated amyloidosis occur systemically, as was found in our patient with the apoA-IV-containing deposits. ApoA-IV is a $46 \mathrm{kDa}$ glycoprotein primarily synthesized by the enterocytes in the small intestine and is important in the absorption, transport, and metabolism of lipids. The plasma concentration varies between 14 and $37 \mathrm{mg} / \mathrm{ml}$, depending on nutritional status. $^{39,40}$ A higher than normal concentration of an amyloid fibril protein precursor often is involved in amyloid pathogenesis. No blood specimens were available from our patient for measurement of this component; however, it is important to note that plasma levels of apoA-IV increase with age. ${ }^{41}$

Although other types of apoA molecules, that is, apoA-I and apoA-II, are known to be amyloidogenic, 
in all systemic cases these components have been the product of mutated genes. ${ }^{5,15-20}$ In contrast, the amino-acid sequence of the apoA-IV fibrils was identical to the corresponding region of the wt protein; furthermore, there were no mutations in the nucleotides of the apoA-IV gene cloned from the patient's genomic DNA.

Characteristically, the systemic amyloidoses are associated with the pathologic deposition of only one biochemical type of amyloidogenic precursor protein, although there has been one report of a case with apoA-I cardiac, laryngeal, and cutaneous amyloidosis in whom TTR also was found in a skin lesion as a minor component. ${ }^{42}$ In contrast, our patient was unique in having two distinct kinds of systemic amyloidosis. We originally had postulated that both types of fibrils were colocated, given that a synthetic apoA-IV peptide spontaneously formed fibrils and enhanced in vitro TTR fibrillogenicity. ${ }^{22}$ Upon further study, we have demonstrated that the apoA-IV and TTR amyloid proteins were deposited independently in anatomically distinct areas within a single organ or blood vessels, as shown immunohistochemically. Further, each could be distinguished from the other on the basis of differences in the extent of Congo red birefringence. Additionally, both types of fibrils had unique ultrastructural morphology.

The finding that TTR and apoA-IV formed discrete deposits and not a diffusely mixed amyloid infiltration is remarkable since both fibril precursors are derived from plasma proteins and not local synthesis. Thus, although amyloid fibrils resemble each other morphologically and in their principle molecular organization, there may be differences that do not allow mixed $\beta$-sheet fibrils. This does not exclude the possibility that fibrils of one protein could have served as a seed or nucleus to promote fibrillogenesis of the second. For example, in vitro studies have shown that $\mathrm{A} \beta$ fibrils can seed IAPP fibril formation. ${ }^{43}$ We can only speculate whether the two amyloid forms described in this paper have developed completely separately, or not. If a seeding mechanism was operating here, it is unlikely that ATTR-fibrils seeded wt apoA-IV since we have not found apoA-IV-related molecules in TTR-containing extracts obtained from over 30 patients with SSA.

Thus, we conclude that our patient had two different forms of systemic amyloid-one composed of apoA-IV and the other of TTR.

\section{Acknowledgements}

We thank Dr JW Kelly for the truncated TTR clone. This study was supported by Swedish Research Council, the Swedish Heart Lung Foundation, FAMY, FAMY-Norrbotten, the AMYL Foundation, the Swedish Society of Medical Research, USPHS Research Grant CA10056 from the National
Cancer Institute, and the Aslan Foundation. AS is an American Cancer Society Clinical Research Professor.

\section{Duality of Interest}

No duality of interest is declared.

\section{References}

1 Westermark P, Benson MD, Buxbaum JN, et al. Amyloid fibril protein nomenclature-2002. Amyloid. J Protein Folding Disord 2002;9:197-200.

2 Merlini G, Westermark P. The systemic amyloidoses: clearer understanding of the molecular mechanisms offers hope for more effective therapies. J Intern Med 2004;255:159-178.

3 Sunde M, Blake C. The structure of amyloid fibrils by electron microscopy and X-ray diffraction. Adv Protein Chem 1997;50:123-159.

4 Sunde M, Serpell LC, Bartlam M, et al. Common core structure of amyloid fibrils by synchrotron X-ray diffraction. J Mol Biol 1997;273:729-739.

5 Andreola A, Bellotti V, Giorgetti S, et al. Conformational switching and fibrillogenesis in the amyloidogenic fragment of apolipoprotein A-I. J Biol Chem 2003;278:2444-2451.

6 Rochet JC, Lansbury Jr PT. Amyloid fibrillogenesis: themes and variations. Curr Opin Struct Biol 2000;10:60-68.

7 Connors LH, Lim A, Prokaeva T, et al. Tabulation of human transthyretin (TTR) variants, 2003. Amyloid: J Protein Folding Disord 2003;10:160-184.

8 Westermark P, Sletten K, Johansson B, et al. Fibril in senile systemic amyloidosis is derived from normal transthyretin. Proc Natl Acad Sci USA 1990;87:28432845.

9 Cornwell III GG, Murdoch WL, Kyle RA, et al. Frequency and distribution of senile cardiovascular amyloid. A clinicopathologic correlation. Am J Med 1983;75:618-623.

10 Cornwell III GG, Sletten K, Olofsson BO, et al. Prealbumin: its association with amyloid. J Clin Pathol 1987;40:226-231.

11 Röcken C, Saeger W, Linke RP. Gastrointestinal amyloid deposits in old age. Report on 110 consecutive autopsical patients and 98 retrospective bioptic specimens. Pathol Res Pract 1994;190:641-649.

12 Matsutani H, Hoshii Y, Setoguchi M, et al. Vascular amyloid of unknown origin and senile transthyretin amyloid in the lung and gastrointestinal tract of old age: histological and immunohistochemical studies. Pathol Int 2001;51:326-332.

13 Johansson B, Westermark P. Senile systemic amyloidosis: a clinico-pathological study of twelve patients with massive amyloid infiltration. Int $\mathrm{J}$ Cardiol 1991;32:83-92.

14 Westermark P, Bergström J, Solomon A, et al. Transthyretin-derived senile systemic amyloidosis: clinicopathologic and structural considerations. Amyloid: J Protein Folding Disord 2003;10(Suppl 1):48-55.

15 Obici L, Bellotti V, Mangione $\mathrm{P}$, et al. The new apolipoprotein A-I variant leu(174) $\rightarrow$ Ser causes hereditary cardiac amyloidosis, and the amyloid fibrils 
are constituted by the 93-residue N-terminal polypeptide. Am J Pathol 1999;155:695-702.

16 Booth DR, Tan SY, Booth SE, et al. Hereditary hepatic and systemic amyloidosis caused by a new deletion/ insertion mutation in the apolipoprotein AI gene. J Clin Invest 1996;97:2714-2721.

17 Benson MD, Liepnieks JJ, Yazaki M, et al. A new human hereditary amyloidosis: the result of a stopcodon mutation in the apolipoprotein AII gene. Genomics 2001;72:272-277.

18 Yazaki M, Liepnieks JJ, Barats MS, et al. Hereditary systemic amyloidosis associated with a new apolipoprotein AII stop codon mutation Stop78Arg. Kidney Int 2003;64:11-16.

19 Nichols WC, Gregg RE, Brewer Jr HB, et al. A mutation in apolipoprotein A-I in the Iowa type of familial amyloidotic polyneuropathy. Genomics 1990;8:318-323.

20 Yazaki M, Liepnieks JJ, Yamashita T, et al. Renal amyloidosis caused by a novel stop-codon mutation in the apolipoprotein A-II gene. Kidney Int 2001;60: 1658-1665.

21 Husebekk A, Skogen B, Husby G, et al. Transformation of amyloid precursor SAA to protein AA and incorporation in amyloid fibrils in vivo. Scand J Immunol 1985;21:283-287.

22 Bergström J, Murphy C, Eulitz M, et al. Codeposition of apolipoprotein A-IV and transthyretin in senile systemic (ATTR) amyloidosis. Biochem Biophys Res Commun 2001;285:903-908.

23 Castano EM, Prelli F, Pras M, et al. Apolipoprotein E carboxyl-terminal fragments are complexed to amyloids $A$ and L. Implications for amyloidogenesis and Alzheimer's disease. J Biol Chem 1995;270: 17610-17615.

24 Puchtler H, Waldrop FS, Meloan SN. A review of light, polarization and fluorescence microscopic methods for amyloid. Appl Pathol 1985;3:5-17.

25 Westermark GT, Johnson KH, Westermark P. Staining methods for identification of amyloid in tissue. Meth Enzymol 1999;309:3-25.

26 Samdal IA, Sletten K, Olsen KE, et al. AL 366-a glycosylated protein of kappa $1 \mathrm{~b}$ origin in a patient with systemic amyloidosis of predominantly nonparenchymatous distribution. Amyloid: J Protein Folding Disord 2001;8:111-114.

27 Hellman U. Sample preparation by SDS/PAGE and ingel digestion. In: Jollés P, Jörnwall H (eds.) Proteomics in Functional Genomics, Vol. 88. BirkHauser Verlag AG: Basel, Switzerland, 2000, pp. 43-54.

28 Schagger H, von Jagow G. Tricine-sodium dodecyl sulfate-polyacrylamide gel electrophoresis for the separation of proteins in the range from 1 to $100 \mathrm{kDa}$. Anal Biochem 1987;166:368-379.
29 Yang CY, Gu ZW, Chong IS, et al. The primary structure of human apolipoprotein A-IV. Biochim Biophys Acta 1989;1002:231-237.

30 Elshourbagy NA, Walker DW, Paik YK, et al. Structure and expression of the human apolipoprotein A-IV gene. J Biol Chem 1987;262:7973-7981.

31 Ponnuswamy PK, Selvaraj S. Structural similarities in the repeat sequences of plasma apolipoproteins, A-I, A-IV, and E. Protein Seq Data Anal 1992;5:47-56.

32 Saito H, Dhanasekaran P, Nguyen D, et al. Domain structure and lipid interaction in human apolipoproteins A-I and E, a general model. J Biol Chem 2003; 278:23227-23232.

33 Anantharamaiah GM, Venkatachalapathi YV, Brouillette CG, et al. Use of synthetic peptide analogues to localize lecithin:cholesterol acyltransferase activating domain in apolipoprotein A-I. Arteriosclerosis 1990;10:95-105.

34 Emmanuel F, Steinmetz A, Rosseneu M, et al. Identification of specific amphipathic alpha-helical sequence of human apolipoprotein A-IV involved in lecithin:cholesterol acyltransferase activation. J Biol Chem 1994;269:29883-29890.

35 Weinberg RB, Jordan MK. Effects of phospholipid on the structure of human apolipoprotein A-IV. J Biol Chem 1990;265:8081-8086.

36 Weinberg RB. Differences in the hydrophobic properties of discrete alpha-helical domains of rat and human apolipoprotein A-IV. Biochim Biophys Acta 1987;918: 299-303.

37 Weinberg RB. Exposure and electronic interaction of tyrosine and tryptophan residues in human apolipoprotein A-IV. Biochemistry 1988;27:1515-1521.

38 Weinberg RB, Spector MS. Structural properties and lipid binding of human apolipoprotein A-IV. J Biol Chem 1985;260:4914-4921.

39 Green PH, Glickman RM, Riley JW, et al. Human apolipoprotein A-IV. Intestinal origin and distribution in plasma. J Clin Invest 1980;65:911-919.

40 Stan S, Delvin E, Lambert M, et al. Apo A-IV: an update on regulation and physiologic functions. Biochim Biophys Acta 2003;1631:177-187.

41 Sun Z, Larson IA, Ordovas JM, et al. Effects of age, gender, and lifestyle factors on plasma apolipoprotein A-IV concentrations. Atherosclerosis 2000;151:381388.

42 de Sousa MM, Vital C, Ostler D, et al. Apolipoprotein $\mathrm{AI}$ and transthyretin as components of amyloid fibrils in a kindred with apoAI Leu178His amyloidosis. Am J Pathol 2000;156:1911-1917.

43 O'Nuallain B, Williams AD, Westermark $\mathrm{P}$, et al. Seeding specificity in amyloid growth induced by heterologous fibrils. J Biol Chem 2004;279:17490-17499. 